\title{
Photoacoustic Based Surface Plasmon Resonance Spectroscopy: An Investigation
}

\author{
K. Sathiyamoorthy, C. Vijayan and V.M. Murukeshan
}

Additional information is available at the end of the chapter

http://dx.doi.org/10.5772/52545

\section{Introduction}

Surface Plasmon resonance effect (SPR) has very important applications in many fields. ${ }^{1-3}$ The most significant of them perhaps is in the field of real-time bio-sensing. It is important for bio-sensors to be sensitive enough to detect even the smallest amounts of bio-molecules. There are several instances when conventional spectroscopy becomes inadequate, even for clearer and transparent materials. Such a situation arises when one attempts to measure a very weak absorption, which in turn involves the measurement of significantly small changes in the intensity of the strong and unattenuated transmitted signal.

Surface plasmons (SP) are the quanta of elementary excitations involving the oscillations of the electron cloud against the background ionic arrangement in a metal surface. SP can be excited at the metal-dielectric interface using light by matching the momenta between the incident photon and the surface plasmon. Momentum matching can be achieved by various techniques. ${ }^{4-7}$ The simplest way to achieve momentum matching between excitation photon and plasmon is utilization of high index prism over the metal medium. SP excitation using prism coupling technique is based on either Kretschmann or Otto configuration. ${ }^{8}$, 9 The present study is based on Kretschmann configuration in which the metal film has direct contact with the prism base. The magnitude of the wavevector of SP depends on the dielectric constants of both the metal and the surrounding dielectric medium. It is extremely sensitive to properties of the dielectric medium which is in contact with the metal. As SP circumvents diffraction, it can be confined in nanosize domain and hence have interaction with material structures of dimension from sub-micrometer to a few nanometers. The most common technique widely examined for investigating the effects of the excitation of SP is the measurement of reflection coefficient of incident optical beam. But such an approach is mostly not suitable for the sample with weak absorption characteristics. Higher detection sensitivities than those possible with such an approach are always desirable for improving 
sensing performance. The measurement of phase variation during SP excitation is expected to provide higher sensitivity to SPR sensors. However, the complexity of the technique associated with phase detection impedes the targeted development. Moreover, most of the optical detection systems (such as linear photo arrays, CCD etc.) currently employed for measuring the reflection remain off the interrogation region due to their sophistication thereby increasing the size and the cost of the system.

The significance of the photo acoustics techniques is in this context that it may pave the way for the design of bio-sensors with greater sensitivity as they are capable of detecting temperature rises of $10^{-6}$ to $10^{-5}$ of a degree in the sample, corresponding to a thermal power generation of about $10^{-6} \mathrm{calories} / \mathrm{cm}^{3}$. Furthermore, since the sample itself constitutes the electromagnetic radiation detector enabling studies over a wide range of optical and electromagnetic wavelengths without the need to change detector systems. Besides, the compactness of the PA detection system enables easy integration with the existing Kretschmann configuration based SPR system for applications such as on-chip detection.

Photoacoustic (PA) spectroscopy is a very powerful analytical tool for examining the optical absorption properties of solids as it measures directly the energy absorbed by the material on exposure to light. Conventional optical absorption/transmission spectroscopy requires a homogenous and partially transparent sample and further, it cannot be used with highly scattering samples, where the scattering affects significantly the accuracy of the measurement of optical absorption coefficient. The only other method of obtaining some spectroscopic information from opaque samples is diffuse optical reflection spectroscopy where special treatment is required for sample surface preparation. Such problems are absent in the case of photoacoustic spectroscopy and spectral information can be obtained from a variety of samples including opaque materials, powders etc.

The photoacoustic effect was first reported by Alexander Graham Bell in 1880 though the wide use of this technique for spectroscopic purposes started only almost after a century. From then, there has been a steady progress in the development of PA spectrometers in view of their potential application particularly in biological and environmental studies. ${ }^{10,11}$ A major improvement in the sensitivity of photoacoustic method has been brought about by the availability of laser sources, highly sensitive microphones and other efficient acoustic detection systems. ${ }^{10-12}$ Markus Nagele et al developed a novel type of highly sensitive multipass resonant PA cell for trace-gas detection making use of new compact low-power laser sources such as quantum-cascade lasers. ${ }^{12}$ Frank Muller et al presented a transportable, highly sensitive photoacoustic spectrometer based on a continuous-wave dual-cavity optical parametric oscillator. ${ }^{13}$ Innovations in the design of inexpensive and high performance photoacoustic spectrometers continue to attract recent attention, rendering this form of spectroscopy increasingly versatile. ${ }^{10,11,14-16}$

The principle and theory of standard PA technique are well established by Rosencwaig who described the fundamental principles and investigated several possible applications of the PA technique. ${ }^{17}$ Patel and Tam reviewed the physics of the technique and extended the technique to many other kinds of applications. ${ }^{18-21}$ The most frequently used methods are 
microphonic detection of the periodic pressure variations developed due to nonradiative deexcitation of the absorbed light by the sample enclosed in an air tight cell. In the case of solid samples, the sample is placed inside a specially designed and acoustically insulated gas filled chamber. The sample is then illuminated with monochromatic light, whose intensity is modulated by means of a mechanical chopper. Light absorption by a material usually leads to two types of deexcitation processes namely radiative and nonradiative. The nonradiative deexcitation processes result in heating the sample. Since the incident radiation is intensity modulated by the chopper, the sample gets heated up repetitively. The resulting periodic heat flow from the solid absorber to the surrounding gas medium creates pressure fluctuations in the cell, which are detected by the microphone. Therefore the depth of specimen responsible for the PA signal is restricted within a thermal diffusion length defined by. ${ }^{22}$

$$
\mu=\sqrt{\frac{k}{\pi \rho f_{c}}}
$$

Where $k$ is the thermal conductivity, $\rho$ the density, $c$ the specific heat and $f$ is the chopping frequency. The effect of scattered light on measurement is completely avoided by placing the microphone at remote place. This high sensitive microphone is connected to the main chamber containing experimental sample through a small tunnel. Thus PAS is immune towards the stray light from the highly scattering sample. The photo-exciting system consists of a high power optical source, a monochromator and light focusing set up. The detection system consists of a high sensitive microphone, preamplifier and a dual phase lock-in amplifier. The uses of lock-in amplifier effectively increases $\mathrm{S} / \mathrm{N}$ ratio and enables the simultaneous measurement of phase and amplitude of the PA signal with respect to light modulation frequency. Other than spectral measurements, the photoacoustic technique can also be used in several other applications such as measurement of thermal diffusivity, detection of phase transition, depth profiling, subsurface imaging etc $[7,8,9,10] .{ }^{23-25}$ The main aspect of cell design is the positioning of the microphone with respect to incident light to avoid scattered light and to improve performance $[11,12,13] \cdot{ }^{26-28}$ In the present study the PA cell designed to study SP excitation and provision is made to study samples of different properties in different gas environments.

Both PA and reflectance techniques are established in view of simultaneous measurement of SP excitation. The conventional way of detection of reflectance is by either a linear array of photodiodes or a goniometer. A single photodetector can also be used to measure reflectance by using a second rotation stage moving in conjunction with rotation of the prism. The system employing these types of configurations is found to be expensive and hence two-prism concepts has been proposed in the recent past. ${ }^{29,} 30$ The aim of the earlier reported two-prism configuration is to avoid above mentioned expensive detection techniques. ${ }^{29}$ The second prism is integrated to counter compensate the displacement that caused due to the beam walk over the metal coupled face of the first prism during SPR angular measurement. ${ }^{29}$ The main aim of usage of second prism in the earlier study is to make the reflected beam stationary and incident normally with respect to a single photodiode over wide angular rotation..$^{29}$ But in the case of SPR measurement, it is imminent 
that the surface plasmon excitation spot i.e., the interrogation spot should remain stationary during SPR angular measurement as the beam walk over the metal face of the prism affects the SPR measurement sensitivity significantly. A two-prism technique with a modified optical configuration is designed to improve SPR measurement. The proposed optical configuration involves two prisms which are positioned in such a way that the SP excitation spot and reflected light remain stationary during angular displacement and rotation. Finally these two concepts such as two prisms configuration and PA measurement are integrated to find application as SPR sensor based on photoacoustic detection

This chapter in this context describes objectives, scope and methodology for this photoacoustic based surface plasmon resonance spectroscopy. The main objective is to carry out photoacoustic investigation of surface plasmons, so as to explore the possibility of employing photoacoustic techniques in real time sensing based on plasmon resonance spectroscopy. Such sensors exploit the high sensitivity of the surface plasmon frequency to the refractive index of layers of adsorbed molecules on the surface. Generally the resonance conditions are influenced by the material adsorbed onto the thin metal film, which will be explored in detail. An in house developed photoacoustic technique is employed as the detection system for spectroscopic investigations. Two types of set-ups are proposed to investigate SP assisted sensing applications. The first set up is based on a closed photoacoustic cell in which dye coated glass and Au substrates used as sensor element. The second sensor is based on photoacoustic based measurement of resonance angle of prismbased SP excitation. The setup is also equipped with photodetecting system to measure the reflectance of the incident light. The applications of two proposed methodologies for possible sensor application are explained by taking porphyrin as sensor element as it finds wide applications in the biomedical field. There has been a lot of recent interest in supramolecular aggregation effects of porphyrins leading to the formation of self organized nanorods, nanowheels and nanotubes in view of their scope in sensor applications as well as the interesting modifications in their physical properties. The use of metalloporphyrin arrays as sensors is a new concept in artificial olfaction. Its application as 'Electronic nose' applications based on visualizing the color changes associated with the interaction of vapors have been reported. Besides, it properties on Au substrate is also investigated to examine the effect of SP on its optical properties and on the improvement of the sensitivity of the measurement.

\section{Review of theory}

\subsection{Theoretical investigation on prism based SP excitation}

The principle of prism based SPR sensor is the measurement of attenuated total reflection (ATR) which is an experimental technique often used with infrared (IR) spectroscopy.

Attenuated total reflection spectroscopy utilizes total internal reflection (TIR) which occurs when a beam of radiation from a denser medium enters a rarer medium above the critical angle $\theta c$. The ATR method in IR spectroscopy involves using a crystal as the high refractive index material to excite the test sample. Infra-red light is passed through the ATR crystal in such a way that it should reflect at least once off the internal surface in contact with the sample. This reflection forms an evanescent wave which extends into the sample, typically 
by a few micrometres. The attenuated energy from each evanescent wave is passed back to the infra-red beam, which then exits the opposite end of the crystal. A detector can be used to measure the exiting beam to detect any changes to the beam.

In the same context SPR sensor also uses a high index prism to excite surface plasmon in the metal film. Here surface plasmon not an evanescent wave as in FT-IR spectroscopy interacts with the test sample but the measurement method is based on same ATR principle. In the SPR sensor, ATR is due to excitation of SP at the SPR angle. It is observed above the critical angle when the momentum of the excitation light matches with that of the surface plasmon i.e., when $k_{s p}=k_{o} n_{p} \sin \theta_{s p}$, where $k_{s p}$ and $k_{o}$ are the wavevectors of the incident photon and excited plasmon respectively, $n_{p}$ is the refractive index of the prism and $\theta$ is the angle of incident beam with respect to normal to the prism surface]. As the excitation of surface plasmon depends on the nature of the surrounding dielectric medium, any change in property of the surrounding dielectric will be reflected as shift in SPR angle.

Fresnel's equations are used to explain the excitation of SP using high index prism. The Fresnel's equations describe how light behaves when it moves through media of differing refractive indices. It describes the reflection and transmission of electromagnetic waves at a dielectric interface. As the SPR experiment setup utilizes an ATR method and the ATR method is applied by using a prism with Kretschmann configuration, where the metal layer is in direct contact with the ATR crystal, Fresnel equations for a three layer model (prism-metal-air) is applicable for the present configuration.

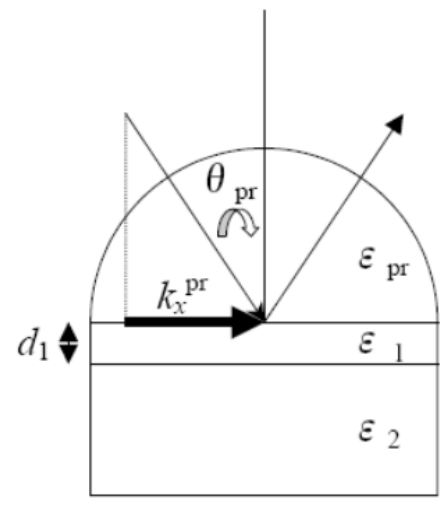

Figure 1. Schematic of three-layer prism setup ${ }^{31}$

Fresnel's equation of three layer model, i.e. prism-metal-air, is defined as

$$
R=\left|r_{p r 12}^{p}\right|^{2}
$$

where $R$ is the reflectance of the incident light, and $r_{p r 12}^{p}$ is the reflected portion of incident light, the superscript $P$ denotes a P-polarized model, and subscript pr12 denotes a prismmedium 1-medium 2 model. 
The reflected portion of incident light is given by ${ }^{31}$

$$
r_{p r 12}^{p}=\frac{r_{p r 1}^{p}+r_{12}^{p} e^{2 i k z 1 d 1}}{1+r_{p r 1}^{p} r_{12}^{p} e^{2 i k z 1 d 1}}
$$

where $r_{p r 1}^{p}$ denotes the reflected potion of light from prism to medium $1, r_{12}^{p}$ denotes the reflected portion of light from medium 1 to medium $2, k z 1 d 1$ denotes the phase factor.

The reflected portion of light from prism to medium 1 is given by ${ }^{31}$

$$
r_{p r 1}^{p}=\frac{\cos \theta_{p r} / n_{p r}-\left(\varepsilon_{1}-n_{p r}{ }^{2} \sin ^{2} \theta_{p r}\right)^{1 / 2} / \varepsilon_{1}}{\cos \theta_{p r} / n_{p r}+\left(\varepsilon_{1}-n_{p r}{ }^{2} \sin ^{2} \theta_{p r}\right)^{1 / 2} / \varepsilon_{1}}
$$

where $\theta_{p r}$ denotes the incident angle of the light entering the prism, $n_{p r}$ denotes the refractive index of the prism, and $\epsilon_{1}$ denotes the dielectric constant of medium 1.

The reflected portion of light from medium 1 to medium 2 is given by ${ }^{31}$

$$
r_{12}^{p}=\frac{\left(\varepsilon_{1}-n_{p r}{ }^{2} \sin ^{2} \theta_{p r}\right)^{1 / 2} / \varepsilon_{1}-\left(\varepsilon_{2}-n_{p r}{ }^{2} \sin ^{2} \theta_{p r}\right)^{1 / 2} / \varepsilon_{2}}{\left(\varepsilon_{1}-n_{p r}{ }^{2} \sin ^{2} \theta_{p r}\right)^{1 / 2} / \varepsilon_{1}+\left(\varepsilon_{2}-n_{p r}{ }^{2} \sin ^{2} \theta_{p r}\right)^{1 / 2} / \varepsilon_{2}}
$$

where $\epsilon_{1}$ denotes the dielectric constant of medium $1, n_{p r}$ denotes the refractive index of the prism, $\theta_{p r}$ denotes the incident angle of the light entering the prism and $\tilde{\epsilon}_{2}$ denotes the dielectric constant of medium 2.

Phase factor is given by(Yamamoto, 2008)

$$
k z 1 d 1=\frac{\omega}{c}\left(\varepsilon_{1}-n_{p r}{ }^{2} \sin ^{2} \theta_{p r}\right)^{1 / 2}
$$

where $\omega=\frac{2 \pi c}{\lambda}$ is the frequency, $c$ denotes the speed of light, $\lambda$ denotes the wavelength of incident light, $\epsilon_{1}$ denotes the dielectric constant of medium $1, n_{p r}$ denotes the refractive index of the prism and $\theta_{p r}$ denotes the incident angle of the light entering the prism.

These equations relate reflectance (intensity of exiting beam) to the refractive indices of the prism, metal and air, thickness of metal layer, wavelength of incident light and the dielectric constants of the metal and air.

\subsection{Theory of photoacoustic spectroscopy}

The PA effect is based on the generation of acoustic signal when a sample enclosed in an airtight chamber is heated periodically on irradiation with an intensity modulated beam. The corresponding pressure fluctuation produced by the sample in the ambient gas is easily detected by a sensitive microphone. Assuming a optically opaque sample and negligible heat flux into the air in contact with the irradiated surface of the sample, the pressure fluctuation inside the chamber is given by 22,32 


$$
Q=\frac{v P_{0} I_{0}\left(\alpha_{s} \alpha_{g}\right)^{1 / 2}}{2 \pi l_{g} T_{0} k_{s} f \sinh \left(l_{s} \sigma_{s}\right)} e^{j\left(\omega t-\frac{\pi}{2}\right)}
$$

where $\gamma$ is the ratio of specific heat capacity of air, $P_{0}$ is the ambient pressure, $T_{0}$ is the ambient temperature, $I_{0}$ is the radiation intensity, $f$ is the modulation frequency, $\alpha_{i}, k_{i} l_{i}$ are thermal diffusivity, thermal conductivity and length of the medium $i=g$ refers to gas and $i=s$ refers to solid sample. The complex quantity $\sigma_{i}=(1+j) a_{i}$, where $a_{i}=\left(\frac{\pi f}{\alpha}\right)^{\frac{1}{2}}$ is the thermal diffusion coefficient of the medium i. The expression in equation (7) can be solved based on whether the sample is thermally thick or thermally thin. A sample is considered to be thermally thick when the heat generated due to the absorption of light in the sample reaches the surface and enters the surrounding air column of the photo acoustic cell. For thermally thick sample, the product $l_{s} a_{s}$ will become greater than $1\left(i . e_{.},\left(l_{s} a_{s} \gg 1\right)\right)$. In that case equation (7) of the PA signal reduces to,

$$
Q=\frac{v P_{0} I_{0}\left(\alpha_{s} \alpha_{g}\right)^{1 / 2}}{\pi l_{g} T_{0} k_{s}} \frac{\exp \left[-l_{s}\left(\frac{\pi f}{\alpha_{g}}\right)^{\frac{1}{2}}\right]}{f} e^{j\left(\omega t-\frac{\pi}{2}-l_{s} \alpha_{s}\right)}
$$

From the above expression it is clear that the photo acoustic signal varies exponentially with the modulation frequency according to $\left.\left[\left(\frac{1}{f}\right) \exp (-b \sqrt{f})\right)\right]$, where $b=l_{s}\left(\frac{\pi}{\alpha_{s}}\right)^{\frac{1}{2}}$ and the phase decreases linearly with $b \sqrt{f}$. Thus, thermal diffusivity $\alpha_{s}$ can be obtained either from the amplitude or phase data with respect to the modulation frequency for the optically opaque and thermally thick sample.

For the thermally thin sample, the product $l_{s} a_{s}$ will become much lesser than 1(i.e., $\left(l_{s} a_{s} \ll\right.$ 1). The equation 7 in this case simplifies to

$$
Q=\frac{v P_{0} I_{0}\left(\alpha_{g}\right)^{1 / 2} \alpha_{s}}{(2 \pi)^{3 / 2} l_{g} l_{s} T_{0} k_{s}} \frac{e^{j(\omega t-3 \pi / 4)}}{f^{3 / 2}}
$$

The above expression implies that the PA signal amplitude from a thermally thin sample under the heat transmission configuration varies as $f^{-1.5}$ and the phase is insensitive to the variation in the modulation frequency. Hence in the case of thermally thin and optically opaque samples, the PA signal is independent of the optical absorption coefficient of the sample, but depends on the wavelength of the incident radiation.

\section{Experimental set-up and characterization}

\subsection{Protocol for sample preparation}

$\mathrm{Fe}(\mathrm{III})$ tetraphenylporphyrin is prepared according to standard procedures reported in literature. ${ }^{33}$ About $50 \mathrm{mg}$ of 5,10,15,20 tetraphenyl porphine Fe(III) chloride procured from 
Aldrich, is dissolved in chloroform and shaken with $1 M$ hydrochloric acid for four hours. The chloroform layer was separated and dried with unhydrous sodium sulphate. The iron porphyrin was purified by recrystallization from chloroform-ethanol mixture and the optical absorption spectrum was checked with standard spectrum. The $\mathrm{HCl}$ treatment is to remove any $\mu$-oxo compounds which are present in iron porphyrin samples. The samples are prepared in different physical forms, namely, liquid, powder and film. The porphyrin solutions are prepared at two different concentrations $10^{-4} \mathrm{M}$ and $10^{-6} \mathrm{M}$ respectively in chloroform. The glass substrate of dimension $1 \mathrm{~cm} \times 1 \mathrm{~cm}$ is ultrasonically cleaned using acetone and then double distilled water a couple of times and vacuum dried. The solution with higher concentration is used to coat a film on the glass plate and Au coated BK7 prism. A few drops of the porphyrin solution are deposited over the glassplate using a $2 \mathrm{ml}$ syringe to form a porphyrin film. Similarly phorphyrin film is formed on Au film coated on the prism base. The deposited film is allowed to dry under vacuum. As the solvent evaporates, the porphyrin film shrinks resulting in the formation islands of porphyrin microclusters on the glass substrate at scattered positions. This approach has been used recently to prepare porphyrin samples where aggregation effects have been observed. ${ }^{34,}{ }^{35}$ Size characterization of the aggregates is done with Atomic Force Microscopy. Contact AFM measurements were done using a Digital Instruments Multimode head attached to a Nanoscope-IV controller. Standard $\mathrm{Si}_{3} \mathrm{~N}_{4}$ cantilevers were used for the normal topography and friction mode imaging. Photoacoustic (PA) studies on powder samples are done by smearing the porphyrin on the grease coated glass substrate.

\subsection{Two-prism configuration to excite and measure SP excitation}

A schematic diagram representing the experimental set-up is shown in figure 2. Laser beam is focused on to the prism base using the lens and reflected light is measured using an ultrasensitive photodiode. The polarizer is set to polarize the incident beam as $\mathrm{P}$ polarized in the direction parallel to the plane of incidence, in this case in the horizontal plane. SP is excited using Kretschmann configuration in which the metal film is in direct contact with the prism base. Lock-in-amplifier and chopper are integrated with the proposed set-up to improve signal to noise ratio (SNR) of the measurement. A chopper is used to modulate the intensity of the incident light and the lock-in amplifier is employed to measure the data with reference to the chopping frequency. Au film is used as the metal film, which is coated on the base facet of BK7 prism.

The prism is fixed on the rotation table and rotated in steps of $0.1^{\circ}$ to change the momentum of incident light with respect to dielectric-metal interface. SP will be excited once the momentum between the incident light and surface plasmon is matched. Maximum energy transfer between photon to surface plasmon takes place at SPR angle and is very sensitive to dielectric property of the material adjacent to $\mathrm{Au}$ film and is given by $K_{s p}=\frac{\omega}{c} \sqrt{\frac{\varepsilon_{m} \varepsilon_{d}}{\varepsilon_{m}+\varepsilon_{d}}}$, where $\omega$ is the frequency, $c$ is the velocity of the light, and $\varepsilon_{m}$ and $\varepsilon_{d}$ are dielectric constants of metal and dielectric medium respectively. 


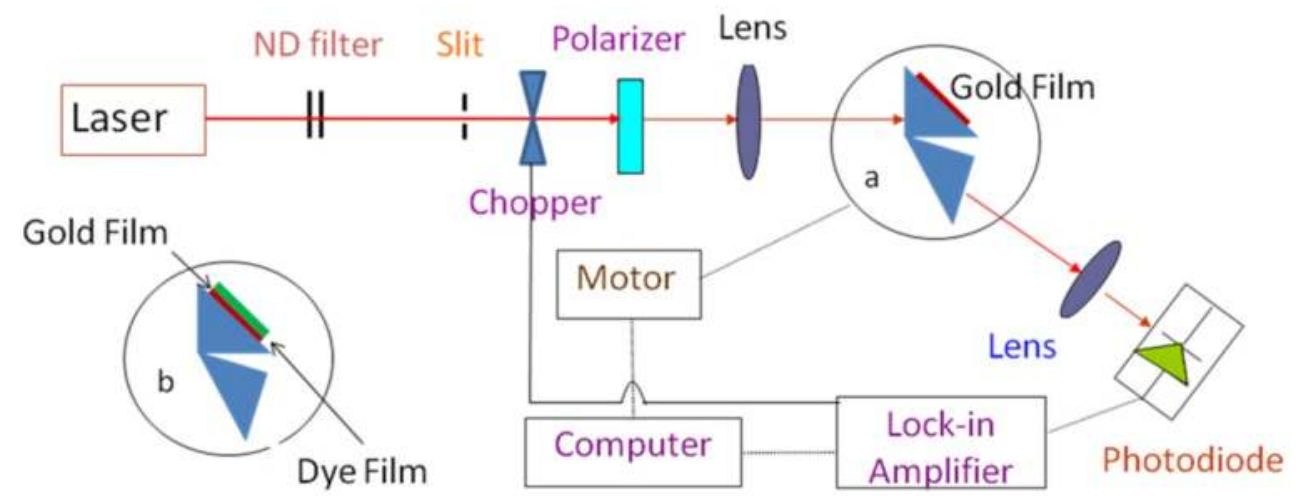

Figure 2. Schematic diagram of experimental set-up for SPR sensor

The point at which SP is excited is called the interrogation point/spot. It is represented by a symbol $S$ in figure 3 . This spot should not walk over the prism base during SPR measurement and is avoided by positioning the prism with respect to rotation axis $(\mathrm{P})$ of the prism table. ${ }^{36}$

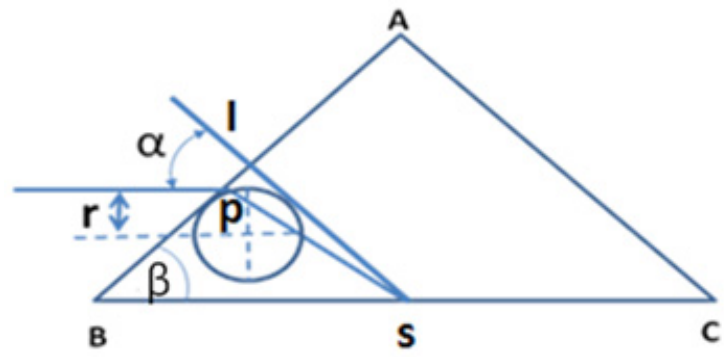

Figure 3. The configuration for incident ray with respect to axis of rotation of the prism table $(\mathrm{P})$ to keep the coupling spot remains practically stationary during SPR measurement

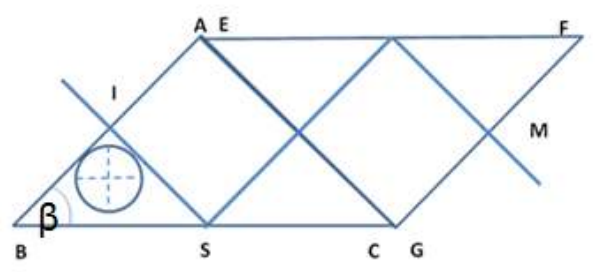

a

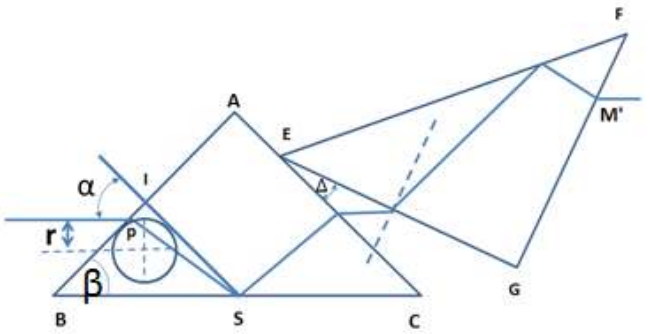

b

Figure 4. The two-prism configuration with (a) angle $=0^{\circ}$ and (b) angle $=\Delta^{\circ}$ between two prisms and ray traces within the prisms 
Figure 3 represents a single prism marked by $\mathrm{ABC}$. The solid line (IS) represents the normal incidence of the excitation beam. The centre of the dotted cross represents the axis of the rotation. A circle is drawn with axis of rotation as center and $r=l_{p} / n_{p}$ radius, where $l_{p}$ is the length of the light path inside the prism from the entrance face to the coupling spot and $n_{p}$ is the refractive index of the prism. It touches the prism surface at I and the beam which strikes at the point of contact (I) always ensures stationary SP excitation spot (S) during SPR angular rotation.

Though the coupling spot remains stationary at the prism base, the reflected light from it always rotates with respect to rotation axis. In such configuration, a linear photodiode array or goniometer is used to collect reflected light from the prism base and is quite expensive. Hence the significance of the concept of employing the two prism configuration. ${ }^{29,}{ }^{30}$ In the two prism technique, the first prism $(\mathrm{ABC})$ is used to excite surface plasmon and the second prism (GEF) is employed to counter the orientation of reflected light from the first prism to opposite direction. This configuration always ensures that the reflected light from both prisms always remains fixed in orientation with respect to the incident beam direction and hence a single photodiode is employed to record the reflected light. Figure 4 represents the two-prism configuration with $\mathrm{ABC}$ and GEF as the two prisms. In figure 4 (a) each prism has been rotated by $90^{\circ}(\Delta=0)$ and the combination act as a parallel plate. The beam which is incident normally (IS) on two prism combination will also emerge out normally at $\mathbf{M}$. Though the interrogation spot remains stationery during rotation, the light getting reflected from the second prism (GEF) will makes a walk-over on the prism base. Hence there will be a small shift $(\delta)$ in the exit beam $\left(\mathbf{M}^{\prime}\right)$ with respect to fixed point $\mathrm{M}$ and the amount of shift depends on angular separation $\Delta$ between the two prisms. Figure 4 (b) represents the shift of the exit beam $\mathrm{M}$ ' due to angular separation $\Delta$ and is given by

$$
\delta=\frac{l}{2} \cos \left(\sin ^{-1}\left(\frac{1}{n_{p}} \sin \left(\frac{1}{n_{p}}\left(\sin \alpha \cos \beta+\left(n_{p}{ }^{2}-\sin ^{2} \alpha\right)^{1 / 2} \sin \beta\right)+\Delta\right)\right)\right)
$$

where $\alpha$ is the angle of rotation of the prism with normal incident angle, $\beta$ is the angle of the prism and $\Delta$ is angle between the prisms, $n_{p}$ is the refractive index and $l$ is the length of the prism facet,

Figure 5 represents the shift $(\delta)$ as a function of incident angle $\alpha$. The plot A represents the displacement curve for the prism configuration $\Delta=0$. It shows minimum at $90^{\circ}$ rotation and exhibits displacement of about $0.035 \mathrm{~mm}$ with respect to $\mathbf{M}$ during the angular rotation between $40^{\circ}$ to $120^{\circ}$ which is found to be lower than the earlier reported two prism configuration. ${ }^{29}$ The shifts in displacement for different angular separations $(\Delta)$ are also investigated. The shift in minimum towards higher degrees due to increase in angular separation $(\Delta)$ is observed. Displacement studies for different angular separations $(\Delta)$ are represented by the graphs $\mathrm{A}, \mathrm{B}, \mathrm{C}$ and $\mathrm{D}$. The shift is about $2^{\underline{o}}$ per $3^{\underline{o}}$ angular separation is observed.

In the present SPR sensor study, the angle $(\Delta)$ between the two prisms is selected as $3^{\underline{o}}$ to get the reflected beam well deviated from the direction of the incident beam for interference free 
detection. Besides this, it is ensured that the reflected light remains well within the active area of the photodiode during the SPR angular detection. Both amplitude and phase of the reflected light are measured simultaneously (figure 6). The figure 6(a) represents the measurement of reflectance as the function of the angular rotation. The dip in the graph is due to excitation of SP and has an SPR angle at $43.6^{\circ}$. The narrowness of the resonance dip indicates the good confinement of SP at the metal-dielectric interface. The experiment is repeated for improved signal to noise ratio and the obtained signal is theoretically analyzed.

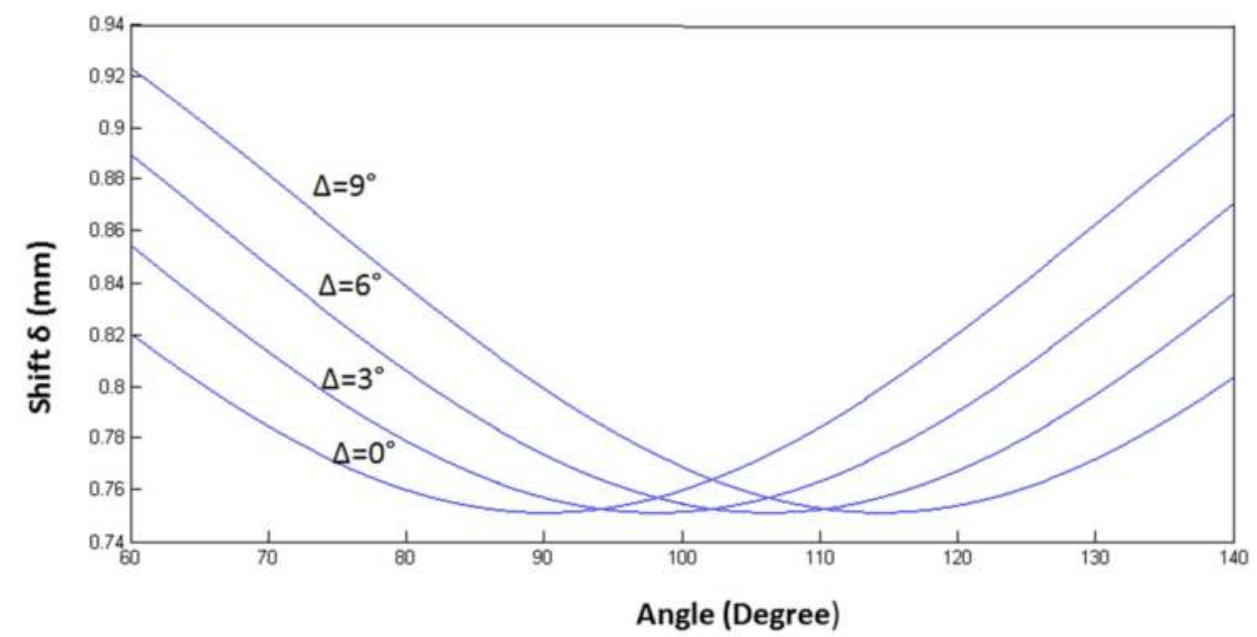

Figure 5. Theoretical simulation of the expression (10), Shift ( $\delta$ ) in terms of Angle $(\alpha)$ for various angular separations $(\Delta)$

The quantitative description of the reflectivity $R$ for the $P$ wave is given by Fresnel's equations for systems comprising three or more layers as,

$$
R=\left|r^{p}{ }_{012}\right|^{2}=\left|\frac{r_{01}^{p}+r^{p}{ }_{12} \exp \left(2 i k_{z 1} d\right)}{1+r^{p}{ }_{01} r_{12}^{p} \exp \left(2 i k_{z 1} d\right)}\right|^{2}
$$

where $d$ is the thickness of the metal film, and the reflection coefficient of the $P$ wave is given by

$$
r_{i k}^{p}=\left(\frac{k_{z i}}{\varepsilon_{i}}-\frac{k_{z k}}{\varepsilon_{k}}\right) /\left(\frac{k_{z i}}{\varepsilon_{i}}+\frac{k_{z k}}{\varepsilon_{k}}\right)
$$

$r^{p}{ }_{i k}$ is the Fresnel coefficient of $p$-polarized light between the $i^{\text {th }}$ layer and the $k^{\text {th }}$ layer in the glass prism(layer 1)/metal (layer 2)/sample (layer 3) configuration (refer figure 1b).

Moreover $r_{012}^{p}=\operatorname{Re}\left(r_{012}^{p}\right)+\operatorname{Im}\left(r_{012}^{p}\right)$. Hence, the phase of the reflected light can be expressed as 


$$
\phi=\tan ^{-1}\left(r_{012}^{p}\right)=\tan ^{-1}\left(\operatorname{Im}\left(r_{012}^{p}\right) / \operatorname{Re}\left(r_{012}^{p}\right)\right)
$$

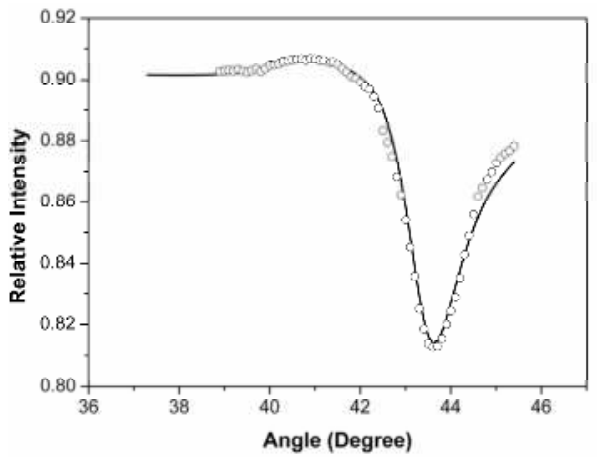

(a)

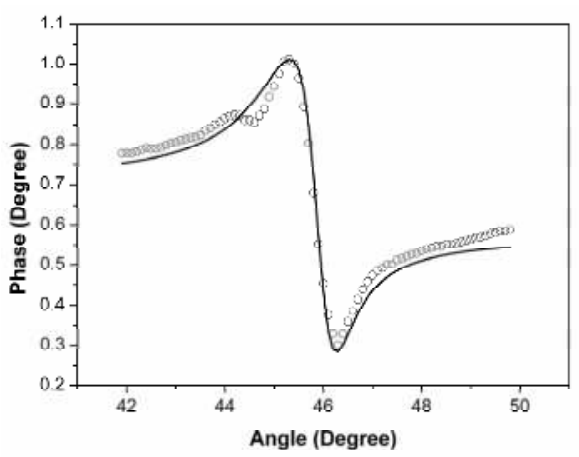

(b)

Figure 6. (a) the reflectivity and (b) the phase shift of the reflected light of a SPR sensor. Circles represent the experimental value and solid lines represent the theoretical simulation

Using the expression (11), theoretical fit on the experimental data (circles) is done to get the refractive index and thickness of the $\mathrm{Au}$ film. The solid line in figure 6(a) represents the theoretical fit. The refractive index and thickness of Au film are found to be0.1726 + i3.3418 and $85 \mathrm{~nm}$ respectively at wavelength $532 \mathrm{~nm}$.

Theoretical simulation is also performed on the obtained phase data (figure $6(\mathrm{~b})$ ) by using expression (13) to validate the results obtained through amplitude data (figure 6(a)). The solid line in figure $6(\mathrm{~b})$ represents the theoretical fit done on the measured phase data pointed represented by circles. It may be noted from figure $6(b)$ that the theoretical fit matches exactly with the experimentally obtained phase data.

\subsection{Variable volume closed photoacoustic cell}

Figure 7 shows a sketch of the designed variable volume closed PA cell and corresponding experimental set-up. The cell is made from an aluminium rod of diameter $80 \mathrm{~mm}$. The main chamber is cylindrical in shape with a volume that can be varied between 160to $640 \mathrm{~mm}^{3}$. The microphone is connected to the main chamber through a narrow tunnel of diameter $1.2 \mathrm{~mm}$. A half inch $50 \mathrm{mV} / \mathrm{Pa}$ sensitive microphone from G.R.A.S is used as PA detecting element. The head of the microphone is acoustically isolated from the surroundings by a proper protective cover made of aluminium, screwed to the side of the PA cell. This cover also prevents dust particles from reaching the microphone diaphragm. PA cell is designed in such a way that its cell volume could be changed to study samples of different properties in different gas environments. The sample holder is made of a brass rod of $1 \mathrm{~cm}$ thick and 1 $\mathrm{cm}$ diameter with a depression on the flat surface. Sample holders with depression of various depths are also made to accommodate samples of different absorption capacities. Particularly samples with low absorption coefficient are taken in relatively large amounts in 
the case of PA studies. Since the PA signal varies inversely as the gas volume and the thermal conductivity of the gas $\left(\sqrt{k_{g}} \frac{P_{0}}{T_{0}}\right.$ where $k_{g}$ is the thermal conductivity of the sample, $P_{0}$ the pressure and $T_{0}$ temperature of the coupling gas medium), the distance between the sample and the cell window must be greater than the thermal diffusion of the gas since the boundary layer of the gas acts as an acoustic piston generating the pressure wave. The distance of the sample surface from the optical widow is varied by screwing the threaded cylindrical rod, on which sample holder rests, connected at the base of the PA cell. (see figure.1). This alters the cell volume, which is an important design parameter. The source used to excite optically the sample is xenon lamp of $500 \mathrm{~W}$ from M. Watnabe \& Co Ltd. An image of the source is condensed within the size of the entrance slit of the monochromator using a proper combination of optical components to maximize the amount of light entering the monochromator to increase intensity of the monochromatised light. Since the intensity of the PA signal is proportional to the intensity of the incident light, the monochromatised light from the exit face of the monochromator is tight focused on the sample surface. The intensity of the light falling on the sample is modulated by a mechanical chopper of frequency $10 \mathrm{~Hz}$ (SR540 chopper) and the reference output of the chopper controller is connected to the lock-in amplifier (SR830 dual-phase digital lock-in amplifier). The lock-in amplifier analyses the PA signal with respect to chopping frequency. The designed PA spectroscopic setup is characterized by measuring frequency response of the spectrometer using carbon black and the result agrees well with the Rosencwaig's theory [figure8]. Then the photoacoustic spectrometer is standardized by recording the spectrum of Holmium oxide. ${ }^{37}$

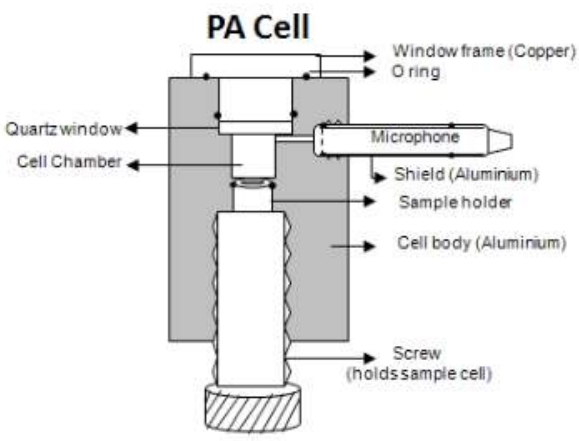

a

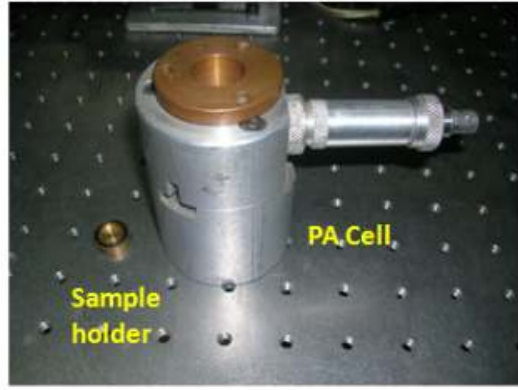

b

Figure 7. (a) Schematic diagram of Photoacoustic cell and (b) Experimental set-up.

The entire PA system is computer interfaced. Wavelength tuning of the monochromator is achieved by a stepper motor rotated by a driving circuit which contains switching circuit powered by $2 \mathrm{~N} 3055$ power transistor and speed of the scanning is controlled through LPT1 port of the computer. The lock-in amplifier is controlled through the RS232 port. 


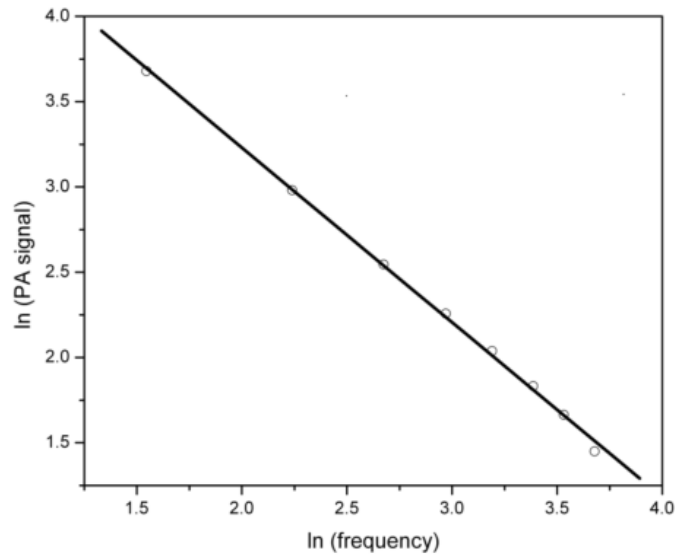

Figure 8. Frequency response of the PA cell.

\subsection{Photoacoustic based surface plasmon resonance spectroscopy}

The photoacoustic set-up to measure resonance angle of SP excitation in prism coupling technique is shown in the following figure. ${ }^{38}$ The light from the source [DPSSL, SUWTECH LDC-1500] was coupled to an optical fibre and guided to an assembly consisting of a polarizer and an optical chopper. Another optical fibre is used to collect the polarised beam to guide through the focusing lens onto the prism base. The prism was mounted on the PA chamber containing microphone. The PA chamber was fabricated in such a way that a small air cell separates the gold-layer and the transducer chamber so as to enable the creation of sound waves.

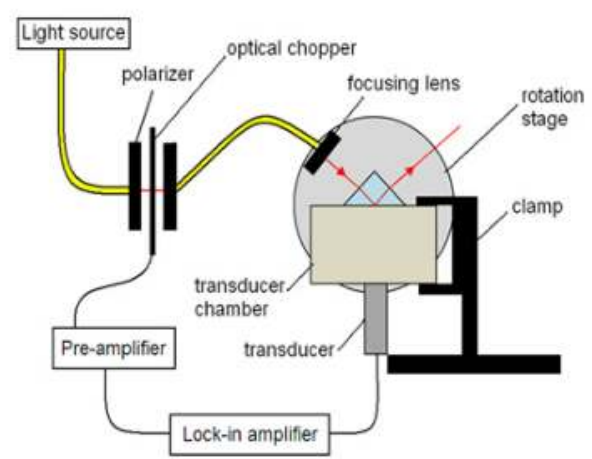

(a)

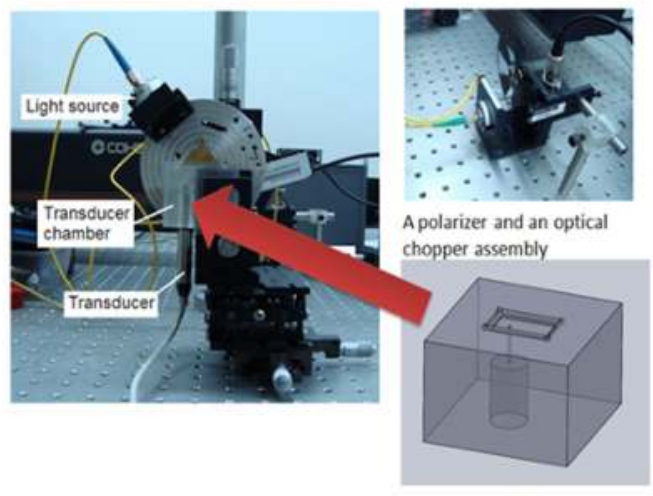

PA Chamber

(b)

Figure 9. (a) Schematic of SPR experiment setup measuring photoacoustic signal (side view) and (b) the corresponding experimental set-up 
The technical drawings for the transducer chamber can be found in figure 9 (b). It encloses chamber of depth $1 \mathrm{~mm}$. PA chamber is connected to the microphone by a tunnel of diameter $1.2 \mathrm{~mm}$ and a length of about $14 \mathrm{~mm}$. The contact areas between the prism and transducer chamber as well as between the transducer chamber and the transducer were sealed using vacuum grease to prevent any leakage of the acoustic piston effect out of the air pocket. The transducer chamber is positioned in such a way that the beam focuses on a stationary spot on the gold nano-layer on the prism throughout the scanning range of incident angles. The beam spot on the gold nano-layer is ensured to be stationary for the same reasons mentioned earlier in SPR experiment conducted for reflectivity measurement. For this investigation, a pre-amplifier with filter is used in addition to the lock-in amplifier to further improve the signal to noise ratio. The transducer is connected to the source input, while the optical chopper is connected to the reference input channels of the lock-in amplifier. The rotation stage is again jogged at intervals of $0.1^{\circ}$, and the photoacoustic signal at each jog step is recorded. Due to the sensitivity of the transducer, Fast Fourier Transform (FFT) filter smoothing was done on the photoacoustic signals to eliminate high frequency noise.

Carbon black is a strong absorber of the light where $(1 / \alpha)<1$ and $\mu>1$ (where $\alpha$ isthe optical absorption coefficient) and hence a strong PA signal is expected. The strength of the signal is inversely proportional to the chopping frequency according to Rosencwaig's theory. ${ }^{22,} 39$ Thus a log-log plot of the PA signal versus chopping frequency is expected to be a straight line of slope-1.

The PA cell is tested by recording the spectrum of carbon black as the standard sample. A Helium-Neon laser is used as the source of excitation. The PA signal is recorded as the function of modulation frequency obtained using a mechanical chopper. Figure 10 represents the measured frequency response of the PA cell. The data fit well to a straight line of slope -1 .

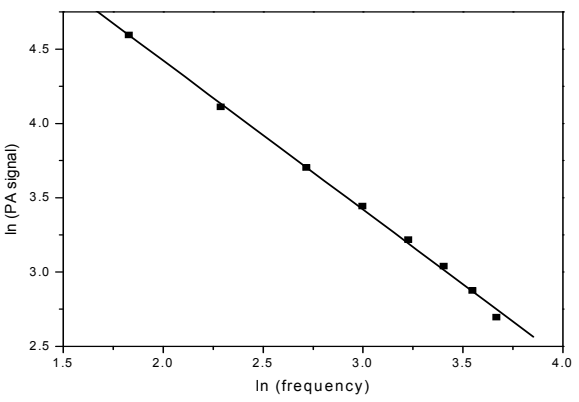

(a)

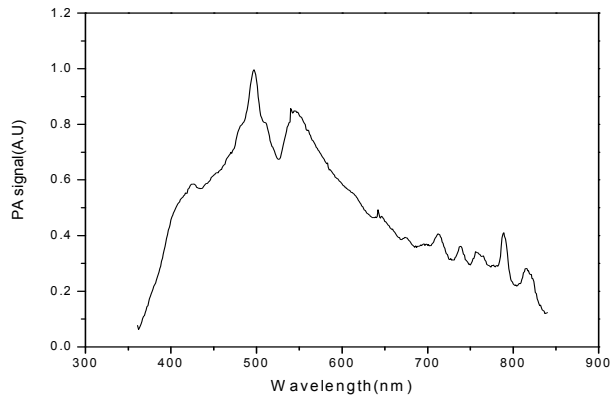

(b)

Figure 10. (a) Frequency response of PA cell and (b) PA Spectrum of carbon black.

Fig 10 (b) shows the PA spectrum of carbon black recorded with the Xenon lamp as the source. In the case of thermally thin and optically opaque samples, the PA signal is 
independent of the optical absorption coefficient of the sample, which in turn depends on the wavelength of the incident radiation. Thus the carbon black sample, which satisfies the above condition, should exhibit the spectrum of the source used. The observed spectrum shows the intensity variation of the xenon lamp as a function of wavelength.

\section{Results and discussion}

The application of the proposed configuration is illustrated by taking porphyrin as sample (dielectric medium). There has been a lot of recent interest in supramolecular aggregation effects of porphyrins in view of their scope in sensor applications. The use of metalloporphyrin arrays as sensors is a new concept in artificial olfaction.

The metalloporphyrin selected for the present study is FeTPPCl. Figure 11 shows the AFM pictures of the film containing FeTPP aggregates on the glass plate. Microclusters of the FeTPP in the size range of 0.8 to 1.6 microns are clearly seen in the picture. The AFM pictures indicate an inhomogeneous distribution of porphyrin molecules obtained on the glass surface.

FeTPP aggregates on the glass plate are studied using optical absorption (OA) and photoacoustic spectroscopy. Figure 12 shows the PA spectrum of FeTPP in three physical forms, namely, solution at low concentration (curve A), powder (curve B) and film on glass plate (curve C). The PA spectrum of the solution at low concentration is obtained by depositing a couple of drops of porphyrin solution on the tissue paper. In the case of powder samples, only a few micrograms are taken to avoid signal saturation. The PA spectrum of aggregates on the glass plate is compared with those of porphyrin solution at low concentration deposited on tissue paper and porphyrin powder in order to distinguish between the effects due to aggregation and concentration.

The Soret band is seen in figure 12 at around $450 \mathrm{~nm}$ and the $\mathrm{Q}$ bands in the region of 500$600 \mathrm{~nm}$. These features are similar to those reported for the solution spectrum. ${ }^{40}$ The peak corresponding to the Soret band is found to be red shifted to $450 \mathrm{~nm}$ in the case of films from the corresponding value of $444 \mathrm{~nm}$ for the spectra of the powder as well as the dilute solution. This shift and the accompanying broadening occur only in the film and not in the cases of the dilute solution. These effects are not observed even in the case of the powder sample where the concentration is high. Thus the observed spectral features appear to result from aggregation effects rather than concentration effects. The aggregates could be Jaggregates which are characterized by shifting of bands to larger wavelengths with respect to long wavelength absorption band of the monomers at about $444 \mathrm{~nm}$. Figure 12(i) shows the OA spectrum of the films on the glass substrate. No specific feature is seen in the $\mathrm{Q}$ band region in the optical spectrum. However, the PA spectrum, shown in figure 12ii(C) clearly exhibits a pronounced two-peak structure between 500-600 nm. Such features are usually attributed to hyper spectra due to metal to ligand charge transfer. ${ }^{33,}{ }^{41}$ These arise from the considerable mixing of the metal $\mathrm{d}_{\pi}$ orbitals with the LUMO of the Porphyrin, which renders Fe porphyrins of considerable interest for photochemical studies. The broadening, almost nonzero extinction coefficient throughout the visible region and spectral shifts of the 
bands are indicative of formation of aggregates during the preparation of films on glass substrate. ${ }^{35}$

The $\mathrm{Q}$ bands in OA spectrum are not well pronounced as compared to PA spectrum of the film, apparently due to the inhomogeneous distribution of film on the glass substrate and low absorption of the sample. The broad spectral features of FeTPP in liquid form at low concentration, film form and powder form appear to be similar. However, the process of preparing a solution-dried film on glass plates leads to some amount of aggregation as judged by the shift and the broadening in the bands. The hyper spectral features in $Q$ band region of the FeTPP are clearly resolved in PA spectra, particularly in the case of aggregates. This could be due to the fact that the efficiency of nonradiative thermal processes is much larger in aggregates (than in monomeric species) to which PAS is very sensitive. The increased metal-ligand energy transfer in the case of aggregate films could be the reason for the observation of well pronounced hyper spectra.

PA studies at low concentration solution and powder are done to bring out the effect of concentration on shifting and broadening of the peaks in PA spectra. The PA spectrum of porphyrin solution at low concentration is studied by depositing a few drops of porphyrin on the tissue paper, which is a convenient way of recording the spectra of liquid samples using the microphone-based PA spectrometer. The experiment is also repeated by depositing low concentrated solution on a glass plate to avoid aggregation. However, such samples did not produce any appreciable PA signal due to the low concentration. Aggregation processes are also absent in powder samples and occur when the porphyrin films are deposited on the glass substrate. The PA spectra reveal the aggregation effects in a more detailed manner compared to OA spectra. PA spectra of powder samples can be obtained using only at a few micrograms of the sample smeared over the grease coated glass plate as signal saturation effects are observed at higher density. ${ }^{42}$

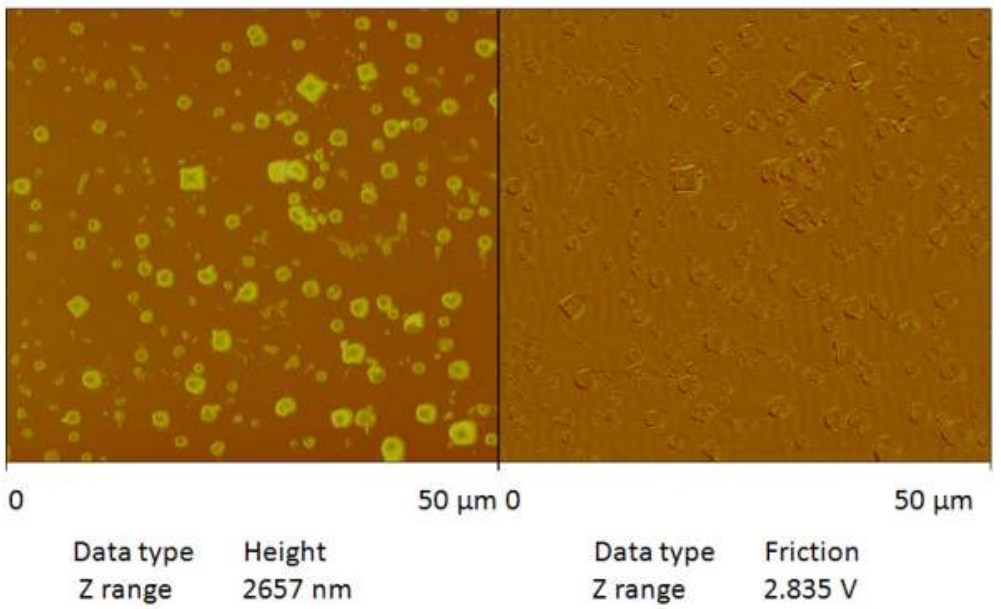

Figure 11. AFM picture of FeTPP aggregates on glass substrate 


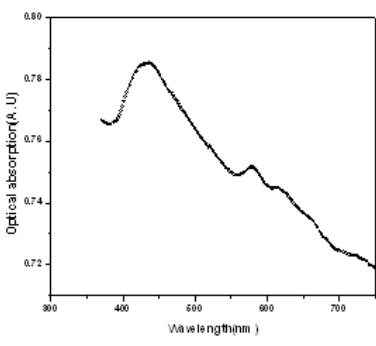

$i$

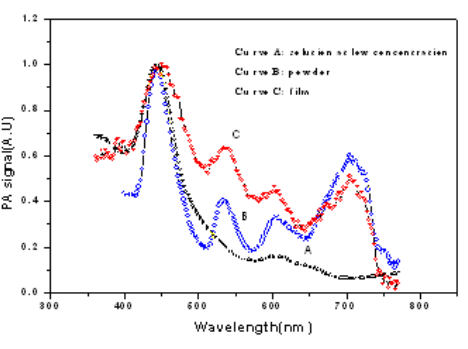

ii

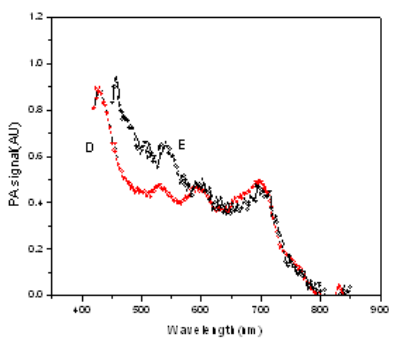

iii

Figure 12. (i) Optical absorption spectrum of FeTPP on glass substrate, (ii) Photoacoustic spectrum of FeTPP.[(a) curve A -solution at low concentration, curve B -powder and curve C- film on glass substrate.] and (iii) Photoacoustic spectrum of FeTPP.[(d) Curve D -dye film on glass plate and curve E -dye film on Au film.]

AFM studies on porphyrin deposited on Au film show no distinct features as observed in the case of porphyrin film formed on glass substrate. PA studies on porphyrin film on $\mathrm{Au}$ substrate \{Figure 12(iii) show improved PA signal as compared to porphyrin film on glass substrate [Figure 12(iii) D]. The proposed concept based on PA cell with porphyrin-glass and porphyrin-Au substrates is expected to find applications as gas sensor.

Investigation of SPR excitation is performed using the two-prism technique. Figure 13shows surface plasmon excitation in Au film of thickness $60 \mathrm{~nm}$ using prism in Kretschmann configuration. The graph is a plot of reflectivity against angular rotation of the prism. It exhibits a dip at 43.95 degrees due to excitation of surface plasmon. The angle at which SP is excited is called surface plasmon resonance angle. From the experimental data, it can be observed that a peak in reflectivity occurs before a dip. At the peak, the ratio of incident light intensity to reflected light intensity is at a maximum. This would mean that there is minimum loss of incident light and the peak is a result of TIR. The peak is at the angle where the highest amount of energy of the incident light is passed, through the evanescent wave along the metal and crystal interface, back to the reflecting light.

Therefore, the subsequent minimum reflectivity would give the SPR angle, since the angle is after the critical angle for TIR, and is where the greatest amount of energy is lost due to maximum excitation of surface plasmons. After the SPR angle, reflectivity increases as incident angle increases. This indicates that the amount of energy lost through surface plasmon excitation is decreasing as the consequence of deviation from momentum match between incident photon and excited SP, with the light intensity returning to initial levels. The profile of reflectivity against incident angle forms a basis for comparison with photoacoustic signals.

The experimental set-up used to study SPR is described in the experimental section titled photoacoustic based surface plasmon resonance spectroscopy. Figure 13 shows the graph of photoacoustic signal obtained against incident angle for gold nano-layer of $60 \mathrm{~nm}$ thickness. The graph contains two photoacoustic profiles for two modulation frequencies such as $7 \mathrm{~Hz}$ 
and $11 \mathrm{~Hz}$ trespectively. From the data, it can be seen that at both frequencies, a peak occurs at the SPR angle. This result is consistent with earlier reported data by different groups. ${ }^{43-46}$ For $7 \mathrm{~Hz}$ modulation frequency, the photoacoustic signal is larger at every incident angle as compared to $11 \mathrm{~Hz}$. These indicate that lower frequencies resulted in larger photoacoustic signals.
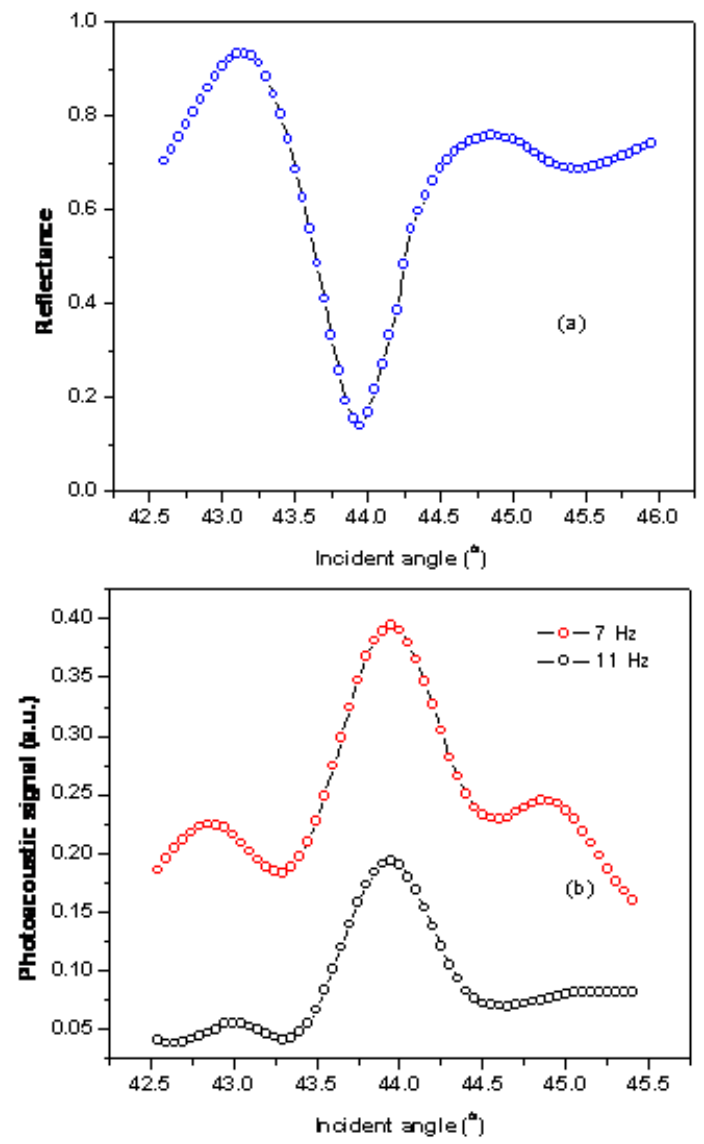

Figure 13. Comparison of (a) reflectivity profile and (b) photoacoustic profile

Figure 13 shows the comparison of the reflectivity profile with photoacoustic signal profile against incident angle. As can be seen from the graph, peaks in reflectivity correspond with dips in photoacoustic signal, and vice versa. It is thus established that reflectivity and photoacoustic signal have an inverse relationship. According to Inagaki et $\mathrm{al}^{43}$, the absorptivity for $p$-polarized photons is affected at each angle of incidence into two parts, one of which contributes to the photoacoustic signal by decaying nonradiatively into heat, and the other part which escapes from the sample by decaying into photons or into emission of photoelectrons without contributing to the photoacoustic signal. 


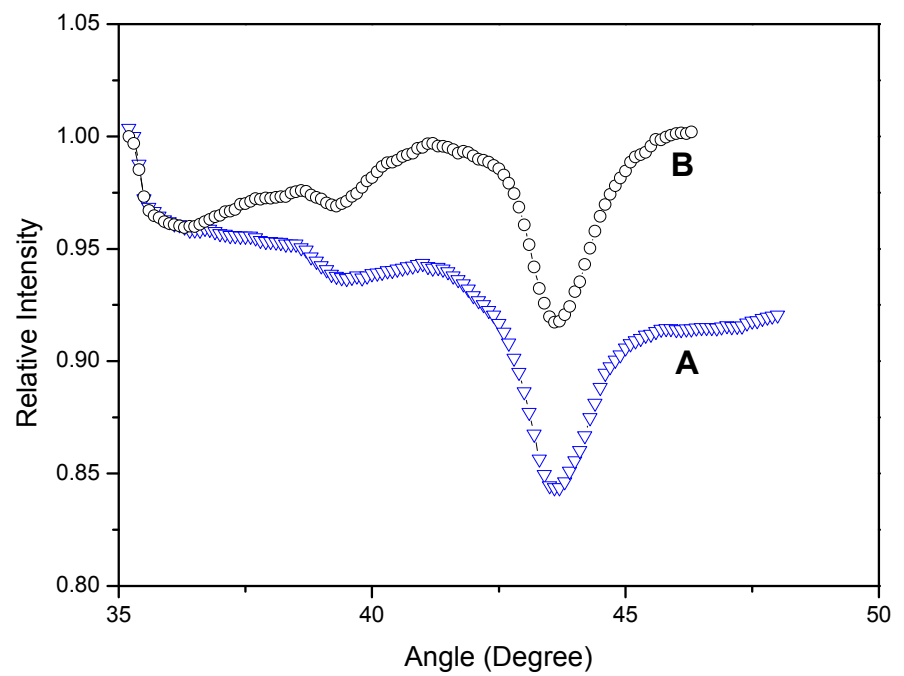

Figure 14. Reflectivity response of a Kretschmann surface plasmon arrangement as a function of angle for $532 \mathrm{~nm}$ wavelength. (A) Bare Au film and (B) Au-Dielectric (porphyrin medium).

At the critical angle where reflectivity is the highest, the absorption of $p$-polarized photons is the lowest since there is little difference between the incident intensity of light and reflected intensity of light. As such, the least amount of $p$-polarized photons decays into heat. Fluctuation of thermal expansion is then at its least, resulting in low amplitudes of the photoacoustic piston effect, i.e. minimal photoacoustic signal. Conversely, At the SPR angle, reflectivity is at its lowest due to maximum absorption of $p$-polarized photons. The resulting amount of p-polarized photons decaying into to heat is at a maximum. This fluctuation of thermal expansion would then be at its maximum, resulting in high amplitudes of the photoacoustic piston effect, i.e. maximum photoacoustic signal.

SPR measurement is repeated on Au-porphyrin dielectric medium to study the response of SPR angle due to adsorption of dye molecules for possible sensor applications. The plot B in Figure 14 represents the corresponding SPR measurement. The result shows a lowering in the reflection minimum of SP excitation as compared bare Au film (plot A in figure 14). The excited surface plasmon in the metallic film suffers significantly from strong damping caused by internal absorption and radiation losses. The damping of surface plasmon is characterized by an exponential decay of its intensity away from the metal surface. The radiative loss is due to plasmon re-emission of light. The metal dissipation loss in this case is given by ${ }^{47}$

$$
\gamma_{m}=\frac{2 \pi}{\lambda_{e}} \frac{\varepsilon_{m}^{\prime \prime}}{\left(\varepsilon_{m}^{\prime}\right)^{2}}\left(\frac{\varepsilon_{m}^{\prime} \varepsilon_{d}^{\prime}}{\varepsilon_{m}^{\prime}+\varepsilon_{d}^{\prime}}\right)^{3 / 2}
$$

where and denote the real and imaginary part of the dielectric constant of the metal, where is the real part of surrounding dielectric medium. This loss can be overcome by gain 
medium such as dye at an optimum concentration. The gain $\gamma_{g}$ needed to compensate for the SP loss is given by ${ }^{47}$

$$
\gamma_{g}=\frac{2 \pi}{\lambda_{e}} \frac{\varepsilon_{d}^{\prime \prime}}{\left(\varepsilon_{d}^{\prime}\right)^{2}}\left(\frac{\varepsilon_{m}^{\prime} \varepsilon_{d}^{\prime}}{\varepsilon_{m}^{\prime}+\varepsilon_{d}^{\prime}}\right)^{3 / 2}
$$

here $\varepsilon_{d}$ is the imaginary part of the dielectric constant of dye medium which is the function of dye concentration $(N)$ and emission cross section $\left(\sigma_{e}\right)$. Hence the lowering in the reflection minimum of SP excitation could be due to dye assisted enhancement of SP.

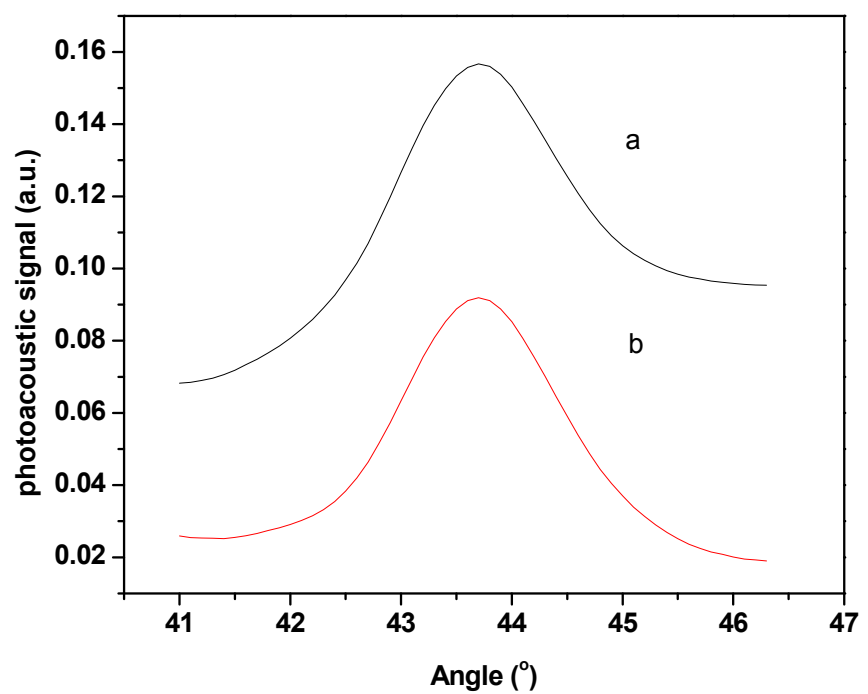

Figure 15. Photoacoustic investigation of (A) Bare Au film and (B) Au-Dielectric (porphyrin medium).

The photoacoustic investigation of SP assisted porphyrin excitation shows a decrease in PA signal (figure 15), which signal could be due to enhanced SP excitation in the presence porphyrin gain medium. The excited fluorophore in the gain medium emits plasmons instead of photons due to strong interaction between the excited dye molecules and electron vibration energy of SP. Any change in the property of the dye due to physio-adsorption and chemisorption will influence the excited SP, which will be reflected in reflective and photoacoustic measurements.

\section{Conclusion}

Photoacoustic investigation of surface plasmons was thus realized, establishing the relationship between SPR and photoacoustics. An in house developed photoacoustic technique was designed and demonstrated to be feasible as a real time sensor based on plasmon resonance spectroscopy through the repeatability of photoacoustic results using 
different modulation frequencies. Theoretical simulations done verify the experimental results for SPR, thereby providing a basis of comparison with the results of the photoacoustic experiment.

There is ample scope for future investigation on realizing photoacoustic bio-sensor of various types. The main aim is the identification of a target molecule by having a suitable biological recognition element attached to the surface of the sensor (be it metal, polymer or glass).

\section{Author details}

K. Sathiyamoorthy and V.M. Murukeshan

Nanyang Technological University, School of Mechanical and Aerospace Engineering, Singapore

C. Vijayan

Department of Physics Indian Institute of Technology Madras Chennai, India

\section{References}

[1] G. Klenkar and B. Liedberg, Analytical and Bioanalytical Chemistry 391, 1679 (2008).

[2] J. Borejdo, Z. Gryczynski, N. Calander, P. Muthu, and I. Gryczynski, Biophysical journal 91, 2626 (2006).

[3] X. Chen, M. C. Davies, C. J. Roberts, K. M. Shakesheff, S. J. B. Tendler, and P. M. Williams, Analytical Chemistry 68, 1451 (1996).

[4] S. Y. Wu, H. P. Ho, W. C. Law, C. Lin, and S. K. Kong, Opt. Lett. 29, 2378 (2004).

[5] Y. H. Joo, S. H. Song, and R. Magnusson, Opt. Express 17, 10606 (2009).

[6] J. D. Wright, C. von Bultzingslowen, T. J. N. Carter, F. Colin, P. D. Shepherd, J. V. Oliver, S. J. Holder, and R. J. M. Nolte, Journal of Materials Chemistry 10, 175 (2000).

[7] L. Pang, G. M. Hwang, B. Slutsky, and Y. Fainman, APPLIED PHYSICS LETTERS 91, 123112 (2007).

[8] Z. Genchev, N. Nedelchev, E. Mateev, and H. Stoyanov, Plasmonics 3, 21 (2008).

[9] H.-S. Leong, J. Guo, R. G. Lindquist, and Q. H. Liu, Journal of Applied Physics 106, 124314 (2009).

[10] S. T. Persijn, E. Santosa, and F. J. M. Harren, Applied Physics B: Lasers and Optics 75, 335 (2002).

[11] J. Li, X. Gao, W. Li, Z. Cao, L. Deng, W. Zhao, M. Huang, and W. Zhang, Spectrochimica Acta Part A: Molecular and Biomolecular Spectroscopy 64, 338 (2006).

[12] M. Nägele, D. Hofstetter, J. Faist, and M. W. Sigrist, Analytical Sciences 17, 497 (2001).

[13] F. Müller, A. Popp, F. Kühnemann, and S. Schiller, Opt. Express 11, 2820 (2003).

[14] L.-y. Hao, J.-x. Han, Q. Shi, J.-h. Zhang, J.-j. Zheng, and Q.-s. Zhu, Review of Scientific Instruments 71, 1975 (2000). 
[15] J. A. Balderas-Lopez, Review of Scientific Instruments 77, 064902 (2006).

[16] J. A. Balderas-Lopez, Review of Scientific Instruments 77, 086104 (2006).

[17] A. Rosencwaig, in Advances in Electronics and Electron Physics, edited by L. Marton (Academic Press, 1978), Vol. Volume 46, p. 207.

[18] C. K. N. Patel and A. C. Tam, Reviews of Modern Physics 53, 517 (1981).

[19] A. C. Tam and C. K. N. Patel, Appl. Opt. 18, 3348 (1979).

[20] C. K. N. Patel and A. C. Tam, Nature 280, 302 (1979).

[21] C. K. N. Patel and A. C. Tam, Applied Physics Letters 34, 467 (1979).

[22] A. Rosencwaig and A. Gersho, Journal of Applied Physics 47, 64 (1976).

[23] K. A. Azez, Journal of Alloys and Compounds 424, 4 (2006).

[24] Q. Shen, M. Inoguchi, and T. Toyoda, Thin Solid Films 499, 161 (2006).

[25] T. S. Silva, A. S. Alves, I. Pepe, H. Tsuzuki, O. Nakamura, M. M. F. d. A. Neto, A. F. da Silva, N. Veissid, and C. Y. An, Journal of Applied Physics 83, 6193 (1998).

[26] N. C. Fernelius, Appl. Opt. 18, 1784 (1979).

[27] L. C. Aamodt, J. C. Murphy, and J. G. Parker, Journal of Applied Physics 48, 927 (1977).

[28] J. W. G. Ferrell and Y. Haven, Journal of Applied Physics 48, 3984 (1977).

[29] B. C. Mohanty and S. Kasiviswanathan, Review of Scientific Instruments 76, 033103 (2005).

[30] K. Sathiyamoorthy, P. A. Kurian, C. Vijayan, and M. P. Kothiyal, edited by R. Guo, S. S. Yin and F. T. S. Yu (SPIE, San Diego, CA, USA, 2007), p. 669818.

[31] M. Yamamoto, Department of Energy and Hydrocarbon Chemistry, Kyoto University, Kyoto-Daigaku-Katsura, Nishikyo-ku, 615-8510, JAPAN 48, 1 (2008).

[32] N. A. George, C. P. G. Vallabhan, V. P. N. Nampoori, and P. Radhakrishnan, Optical Engineering 41, 251 (2002).

[33] F.-P. Montforts, Angewandte Chemie International Edition 43, 5431 (2004).

[34] M. L. Merlau, W. J. Grande, S. T. Nguyen, and J. T. Hupp, Journal of Molecular Catalysis A: Chemical 156, 79 (2000).

[35] N. A. Rakow and K. S. Suslick, Nature 406, 710 (2000).

[36] R. Ulrich and R. Torge, Appl. Opt. 12, 2901 (1973).

[37] K. Sathiyamoorthy, C. Vijayan, and M. P. Kothiyal, Review of Scientific Instruments 78, 043102 (2007).

[38] K. Sathiyamoorthy, J. Joseph, C. J. Hon, and M. V. Matham, edited by M. F. Costa (SPIE, Braga, Portugal, 2011), p. 80010K.

[39] A. Rosencwaig, Optics Communications 7, 305 (1973).

[40] V. Lepentsiotis and R. van Eldik, Journal of the Chemical Society, Dalton Transactions, 999 (1998).

[41] K. S. Suslick, F. V. Acholla, and B. R. Cook, Journal of the American Chemical Society 109, 2818 (1987).

[42] J. W. Lin and L. P. Dudek, Analytical Chemistry 51, 1627 (1979).

[43] T. Inagaki, K. Kagami, and E. T. Arakawa, Appl. Opt. 21, 949 (1982). 
[44] T. A. El-Brolossy, T. Abdallah, M. B. Mohamed, S. Abdallah, K. Easawi, S. Negm, and H. Talaat, The European Physical Journal - Special Topics 153, 361 (2008).

[45] S. Negm and H. Talaat, Solid State Communications 84, 133 (1992).

[46] Q. Shen and T. Toyoda, Jpn. J. Appl. Phys. 39, 511 (2000).

[47] J. Seidel, S. Grafström, and L. Eng, Physical Review Letters 94, 177401 (2005). 